\title{
Hadronic atoms in effective field theory and related aspects
}

\author{
Ulf-G. Meißner ${ }^{a} b$ \\ ${ }^{a}$ Universität Bonn, HISKP (Th), D-53115 Bonn, Germany

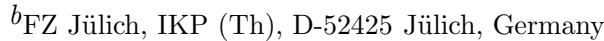

In this talk, I discuss recent work on the extraction of meson-nucleon scattering lengths from the complex energy shifts in pionic deuterium and in kaonic hydrogen in the framework of effective field theories. Also, a simple model for the kaon-alpha-particle scattering length is presented and consequences concerning the possible binding of antikaons in nuclei are discussed.

\section{Introductory remarks}

Hadronic atoms are QED bound states composed of hadrons. They exist in many species, such as pionium $\left(\pi^{+} \pi^{-}\right)$, pionic and kaonic hydrogen $\left(\pi^{-} p, K^{-} p\right)$, pionic deuterium $\left(\pi^{-} d\right)$, and many others. In most cases, the Bohr radius of these atoms is much larger than the typical scale of the strong interactions (QCD) and the average momenta of the hadrons are very small. This allows for a non-relativistic treatment of these composite systems. QCD leads to observable effects in the spectrum of these atoms, most notably the groundstate energy shift $\Delta E$ (with respect to the pure QED value) and the decay width $\Gamma$ of the ground state. One thus has access to hadron scattering at zero energy, that is to the much thought after S-wave hadron-hadron scattering lengths. These are very sensitive to the chiral and isospin symmetry breaking in QCD. Hadronic atoms can be analyzed systematically and consistently in the framework of low-energy Effective Field Theory (EFT), including virtual photons. In this contribution, recent results on the description of pionic deuterium and kaonic hydrogen are discussed. Also of interest are possible deeply bound antikaonic nuclei. In this context, I discuss some very recent work on the S-wave kaon- $\alpha$-particle scattering lengths and its possible extraction at the Jülich COoler SYnchrotron. Note that because of limited space reference is only given to work with direct connection to the material presented in the next three sections. Many more references can be found in the other fine contributions to these proceedings. 


\section{Analysis of pionic deuterium}

First, I briefly review the work presented in [1], where we systematically investigate pionic deuterium within the framework of nested effective field theories (EFTs). To leading order, the standard Deser-type formula for the complex energy shift of the $1 s$ level applies

$$
-\Delta E_{1 s}+i \frac{\Gamma_{1 s}}{2}=\frac{4 E_{1 s}}{r_{B}} a_{\pi d},
$$

and the complex pion-deuteron scattering length has been measured precisely at PSI [2]

$$
a_{\pi d}=(-0.0261( \pm 0.0005)+i 0.0063( \pm 0.0007)) M_{\pi}^{-1} .
$$

The pion-deuteron scattering length gives access to the small isoscalar pion-nucleon scattering length $a_{+}$since $a_{\pi d}=\left(a_{\pi^{-} p}+a_{\pi^{-} n}\right)+$ double scattering + other corrections $=$ $2 a_{+}+\ldots$. Since $a_{+} \simeq 0$, one has to be able to calculate the corrections accurately and systematically - an ideal playground for EFT. While there have already been many studies to relate the deuteron with the nucleon scattering lengths (just to mention a few: multiple scattering, potential model and hybrid approaches, EFT with light, perturbative and heavy pions, and so on), no clear picture concerning the theoretical uncertainty has so far emerged, and that is precisely the main issue here. The whole treatment of this problem naturally falls into several steps, which are analyzed utilizing nested EFTs.

As the first step, one has to consider the extraction of the $\pi d$ scattering length from the $3 p-1 s$ transition energy and width in the pionic deuterium. The next-to-leading order result has been obtained for the level energies, which can be used for an accurate determination of the $\pi d$ threshold scattering amplitude from experimental measurements, see table $1 \mathrm{in}$ [1]. Since the isospin-breaking corrections in this amplitude are not expected to be relevant, given the relatively large theoretical uncertainty in connecting $a_{\pi d}$ with the $\pi N$ scattering lengths (see below), these corrections have been neglected for the time being. Our results essentially confirm the ones earlier obtained by the PSI-ETHZ collaboration [2], we have updated the nuclear and pion finite size corrections utilizing more recent determinations of the respective charge radii.

The main focus of our work [1] is on investigating the possibility to relate $a_{\pi d}$ to the $\pi N$ scattering lengths and on the analysis of the systematic theoretical error in such a procedure. We give a consistent treatment of the problem within the framework of heavy pion (HP) EFT, where the expansion parameter is given by the quantity $x=\gamma / M_{\pi} \simeq 1 / 3$, where $\gamma \simeq 45 \mathrm{MeV}$ is the characteristic bound-state momentum in the deuteron. It was observed in Ref.[3] that in the description of threshold pion-deuteron scattering processes, diagrams with virtual pion absorption/emission are suppressed, i.e. that hadron number is conserved. This can be described most economically by considering the pions as heavy, which leads to HP EFT (or the modified power counting in the chiral perturbation theory approach with light pions, see [4]). To leading order in $x$, one obtains the standard relation

$$
a_{\pi d}^{(0)}=\frac{1+M_{\pi} / m}{1+M_{\pi} / 2 m} 2 a_{+}+\mathcal{O}(x),
$$

with $m$ the nucleon and $M_{\pi}$ the charged pion mass. Note that in this formula $a_{+}$is given to all orders in the chiral expansion. In [1], we have evaluated contributions to 
the quantity $a_{\pi d}$ up-to-and-including $O(x)$, corresponding to next-to-leading order in HP EFT. This gives

$$
a_{\pi d}^{(1)}=-\frac{m\left(1+M_{\pi} / m\right)^{2}}{\pi\left(1+M_{\pi} / 2 m\right)} x a_{-}^{2}\left[g_{a b} \ln \frac{m E_{D}}{\mu^{2}}+J_{a b}\right]+\frac{M_{\pi}^{4}}{4 \pi^{2}\left(1+M_{\pi} / 2 m\right)} x^{3} f_{0}^{r}(\mu),
$$

where (for clarity) the small contributions $\sim a_{+}^{2}$ have been neglected. Here, $E_{D} \simeq$ $2.22 \mathrm{MeV}$ is the magnitude of the deuteron binding energy and $\mu$ the scale of dimensional regularization. Further, $g_{a b}=0.747$ and $J_{a b}=-1.507$ are contributions from one-loop integrals and we recover the standard result in the static limit $M_{\pi} / m \rightarrow 0$. The last term in Eq. (3) is the contribution from the leading four-nucleon-two-pion (three-body) operator. We note that the LEC $f_{0}^{r}(\mu)=\mathcal{O}\left(1 / x^{2}\right)$ is not of natural size. Renormalization group invariance of the scattering length, $d a_{\pi d}^{(1)}(\mu) / d \mu=0$, leads to the following scale dependence of the LEC $f_{0}$,

$$
\mu \frac{d}{d \mu} f_{0}^{r}(\mu)=-\frac{8 \pi\left(1+M_{\pi} / m\right)^{2}}{E_{D} M_{\pi}^{2}} a_{-}^{2} g_{a b} .
$$

In the absence of a determination of $f_{0}$, one can use the scale-dependence of this contribution for estimating the theoretical error in our calculations. This scale dependence is shown in Fig. 1. The scale must be chosen to be of order of the pion mass, but is otherwise arbitrary. In our opinion, the range $100 \mathrm{MeV}<\mu<250 \mathrm{MeV}$ can be roughly considered as a "natural" choice of this scale. It should be also taken into account that the plot in this figure, which corresponds to the recent experimental measurements of the pionic hydrogen decay width, still does not include the isospin-breaking corrections from chiral perturbation theory (CHPT) [5, 6]. As we see from Fig. 1, the theoretical uncertainty due to the unknown LEC $f_{0}$ is rather large. This was already observed in a different setting in [7]. Our main conclusion, concerning the accuracy limits in the extraction of the $\pi N$ scattering lengths from the pion-deuteron data, can be formulated as follows: by far the largest source of uncertainty is the low-energy constant $f_{0}$, which is the genuine short-distance three-body contribution and should be either determined by other experiments or should be obtained by lattice simulations. In particular, one might attempt to get at least the order-of-magnitude estimate from the process $N N \rightarrow N N \pi \pi$ or from the pion-nucleus optical potential, in case of non-equal proton and neutron densities, where $f_{0}$ should be present apart from the "conventional" terms. Neither of these methods seems easy to be applied. But, without having fixed the value of $f_{0}$ at a sufficient precision, it is impossible to improve the accuracy of the prediction of the pion-deuteron scattering length. However, as detailed in [1], it is feasible to estimate $f_{0}$ by using the resonance saturation hypothesis (see appendix $\mathrm{C}$ in that paper). At present time, however, the parameters of the resonance Lagrangian are not known at a sufficient precision, and more effort is needed to pin them down accurately from the experimental data.

Two further remarks are in order. First, in the presence of the absorptive channels, the coupling constant $f_{0}$ is not real. The imaginary part of the $\pi d$ scattering length is given by

$$
\operatorname{Im} a_{\pi d}=\frac{\mu_{d}}{2 \pi} \Phi_{0}^{2} \operatorname{Im} f_{0}
$$




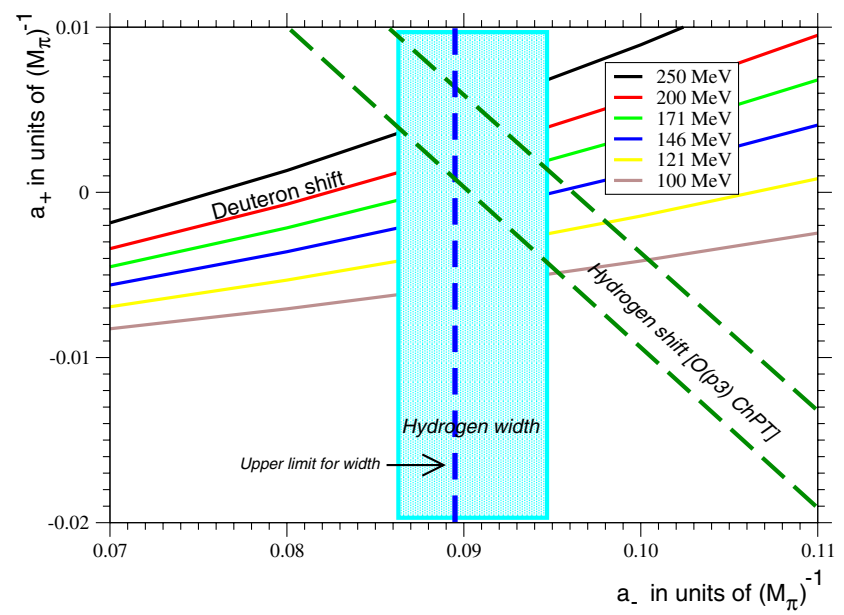

Figure 1: The constraints on the $S$-wave $\pi N$ scattering lengths $a_{+}$and $a_{-}$from the pionic deuterium energy shift for different values of the scale parameter $\mu$. The upper and lower lines correspond to $\mu=250 \mathrm{MeV}$ and $\mu=100 \mathrm{MeV}$, respectively. In addition, we display the constraints from the most recent measurement of the pionic hydrogen shift and width [8]. The upper limit for the width is measured in the $4 p-1 s$ transition in pionic hydrogen.

where $\Phi_{0}^{2}=\gamma^{3} / 2 \pi$ denotes the square of the deuteron wave function at the origin. Further, the imaginary part of the constant $f_{0}$ is directly related to the inelastic channels, which are "shielded" when constructing the HP EFT. The QCD contribution (as denoted by the superscript "str") can be readily evaluated to leading order, it is given by

$$
\operatorname{Im} f_{0}^{\mathrm{str}}=\frac{m p^{\star}}{4 \pi}\left|T\left(p n \pi^{-} \rightarrow n n\right)\right|^{2}, \quad p^{\star}=\sqrt{m M_{\pi}}+\cdots,
$$

with $T\left(p n \pi^{-} \rightarrow n n\right)$ the pion absorption amplitude at threshold. To get the complete imaginary part, which also includes the effect of the $\gamma n n$ state, one writes $\operatorname{Im} a_{\pi d}=$ $\operatorname{Im} a_{\pi d}^{\text {str }}\left(1+1 / R_{\gamma}\right)$, where $R_{\gamma}=2.83$ is the Panofsky ratio. Second, in [1] we have also investigated in detail the differences between the Weinberg approach and the HP EFT. It was shown that, despite the very mild cutoff dependence in the Weinberg approach [4], the uncertainty due to the unknown LECs is significant and is of the same order of magnitude as in the HP EFT. The reason for this is that the large initial- and finalstate nucleon-nucleon interactions lead to the amplification of the initially small LEC contribution. Taking into account this amplification, the theoretical predictions within both approaches are essentially the same. For further discussion of pionic deuterium and related issues, see also the talks by Ericson, Gotta and Simons at this conference. 


\section{Analysis of kaonic hydrogen}

Let us now consider kaonic hydrogen. Its salient features are: i) it can decay into various strong final states $\left(K^{-} p \rightarrow \pi^{0} \Lambda, \pi^{ \pm} \Sigma^{\mp}, \ldots\right)$ and weaker electromagnetic ones $\left(K^{-} p \rightarrow\right.$ $\left.\gamma \Lambda, \gamma \Sigma^{0}, \ldots\right)$. This together with the closeness of the $\Lambda(1405)$ resonance, presumably a meson-baryon bound state, leads to a complicated and interesting analytical structure of the S-matrix in the vicinity of the threshold; ii) the average momentum is very small, $\left\langle\vec{p}^{2}\right\rangle=\alpha \mu \simeq 2 \mathrm{MeV}$ (with $\mu$ the reduced mass and $\alpha$ the fine-structure constant), i.e. the system is highly non-relativistic; iii) the Bohr radius is $r_{B}=1 /(\alpha \mu) \simeq 100 \mathrm{fm}$; iv) the binding energy is $E_{1 s}=\frac{1}{2} \alpha^{2} \mu+\ldots \simeq 8 \mathrm{keV}$ and the width $\left.\Gamma_{1 s} \simeq 250 \mathrm{eV} \ll E_{1 s} ; \mathrm{v}\right)$ since $\mathcal{M}=m_{n}+M_{\bar{K}^{0}}-m_{p}-M_{K^{-}}=5.26 \mathrm{MeV}>0$, there are large unitarity corrections in the relation between the ground state shift and width and the S-wave kaon-nucleon scattering length; and vi) isospin breaking can be systematically included in terms of the small parameter $\delta \sim \alpha \sim\left(m_{d}-m_{u}\right)$. In such a counting, the leading (next-to-leading) order energy shift is $\sim \delta^{3}\left(\sim \delta^{4}\right)$. In what follows, I will discuss the calculation of the energy levels of kaonic hydrogen at next-to-leading order (NLO) in this small parameter.

The calculation of the electromagnetic energy levels and the strong shift is based on the standard non-relativistic Lagrangian (for details see [9] and also the talk by Korobov). Besides kinetic terms, it contains local operators with protons and charged kaons coupled to photons and additional operators with two nucleon and two kaon fields. The coefficients of the hadron-photon operators can be read off from the matching conditions for the kaon and nucleon electromagnetic form factors. The four-hadron operators have complex coefficients. Matching to the relativistic scattering amplitude allows one to express the strong energy shift in terms of the threshold amplitude, i.e. the kaon-nucleon scattering lengths, $a_{0}$ and $a_{1}$, respectively. This leads to:

$$
\begin{aligned}
\Delta E_{n}^{s}-\frac{i}{2} \Gamma_{n} & =-\frac{\alpha^{3} \mu_{c}^{3}}{2 \pi M_{K^{+}} n^{3}} \mathcal{T}_{K N}\left\{1-\frac{\alpha \mu_{c}^{2}}{4 \pi M_{K^{+}}} \mathcal{T}_{K N}\left(s_{n}(\alpha)+2 \pi i\right)+\delta_{n}^{\mathrm{vac}}\right\} \\
\mathcal{T}_{K N} & =4 \pi\left(1+\frac{M_{K^{+}}}{m_{p}}\right) \frac{1}{2}\left(a_{0}+a_{1}\right)+O(\sqrt{\delta}) \\
s_{n}(\alpha) & =2\left(\psi(n)-\psi(1)-\frac{1}{n}+\ln \alpha-\ln n\right.
\end{aligned}
$$

with $\mu_{c}$ the reduced mass of the $K^{-} p$ system and $\Psi(x)=\Gamma^{\prime}(x) / \Gamma(x)$. Further, $a_{0}$ and $a_{1}$ are the isoscalar and isovector S-wave kaon-nucleon scattering length, respectively. The last term in Eq. (8) is the vacuum polarization contribution, which formally is of order $\alpha^{5}$, but amplified by powers of $\mu_{c} / m_{e}$, with $m_{e}$ the electron mass. Therefore, one counts the quantity $\alpha \mu_{c} / m_{e}$ as $\mathcal{O}(\delta)$ and thus vacuum polarizations starts to contribute at leading order $\delta^{3}$. While this representation is correct, it is not accurate enough, precisely because of the large unitarity corrections, which are formally of order $\sqrt{\delta}$. In addition, there are large Coulomb corrections $\sim \alpha \ln \alpha \sim \delta \ln \delta$. As it turns out, by resumming the fundamental meson-baryon bubble to account for the unitary cusp, the large corrections of $\mathcal{O}(\sqrt{\delta})$ can be expressed in terms of $a_{0}$ and $a_{1}$, i.e.

$$
\mathcal{T}_{K N}^{(0)}=4 \pi\left(1+\frac{M_{K^{+}}}{m_{p}}\right) \frac{\frac{1}{2}\left(a_{0}+a_{1}\right)+q_{0} a_{0} a_{1}}{1+\frac{q_{0}}{2}\left(a_{0}+a_{1}\right)}, \quad q_{0}=\sqrt{2 \mu_{0} \Delta \mathcal{M}},
$$




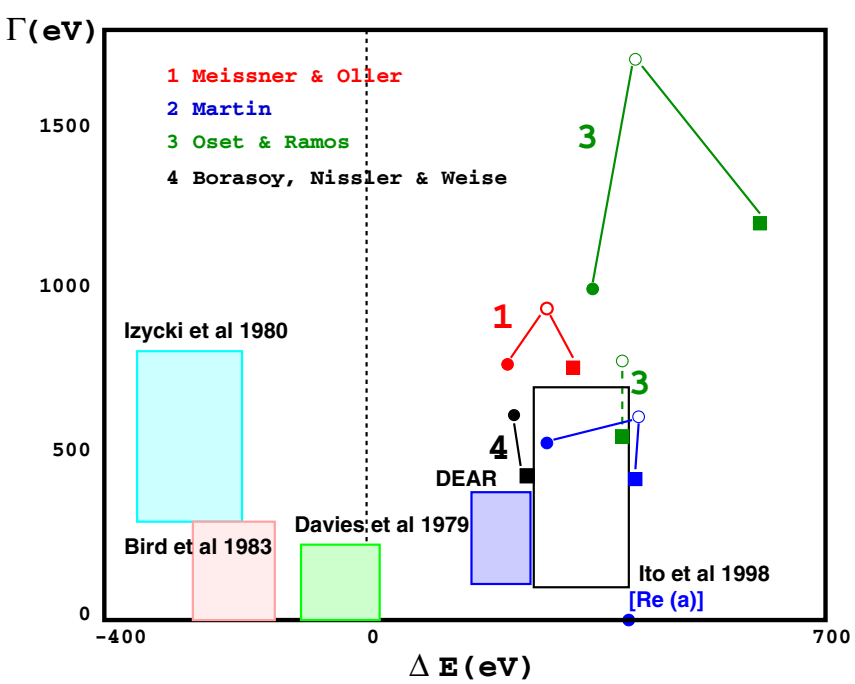

Figure 2: Predictions of the ground-state strong shift $\Delta E_{1}^{s}$ and width $\Gamma_{1}$. Filled circles correspond to using the Deser formula (8), empty circles to using $\mathcal{T}_{K N}^{(0)}$ instead of $\frac{1}{2}\left(a_{0}+a_{1}\right)$ in this formula, and filled boxes to our final formula (12) with $\delta \mathcal{T}_{K N}=\delta_{n}^{\mathrm{vac}}=0$.

with $\mu_{0}$ the reduced mass of the $\bar{K}^{0} n$ system. This result is in agreement with the early one of Dalitz and Tuan [10]. All further corrections of order $\mathcal{O}(\sqrt{\delta})$ collected in the additional term $\delta \mathcal{T}_{K N}$ are expected to be small, $\mathcal{T}_{K N}=\mathcal{T}_{K N}^{(0)}+\frac{i \alpha \mu_{c}^{2}}{2 M_{K^{+}}}\left(\mathcal{T}_{K N}^{(0)}\right)^{2}+\delta \mathcal{T}_{K N}+\mathcal{O}(\delta)$. Note that this representation also incorporates the abovementioned large Coulomb corrections. Putting pieces together, we arrive at the final formula to analyze the older KEK and more recent DEAR data on kaonic hydrogen

$$
\Delta E_{n}^{s}-\frac{i}{2} \Gamma_{n}=-\frac{\alpha^{3} \mu_{c}^{3}}{2 \pi M_{K^{+}} n^{3}}\left(\mathcal{T}_{K N}^{(0)}+\delta \mathcal{T}_{K N}\right)\left\{1-\frac{\alpha \mu_{c}^{2} s_{n}(\alpha)}{4 \pi M_{K^{+}}} \mathcal{T}_{K N}^{(0)}+\delta_{n}^{\mathrm{vac}}\right\}
$$

The following remarks are in order: i) The corrections of $\mathcal{O}(\sqrt{\delta})$ and $\mathcal{O}(\delta \ln \delta)$ are parameter-free (expressed entirely in terms of the scattering lengths) and are numerically by far dominant; ii) we have estimated $\delta \mathcal{T}_{K N}$ in CHPT and found $\delta \mathcal{T}_{K N} / \mathcal{T}_{K N}=$ $(-0.5 \pm 0.4) \cdot 10^{-2}$ at $O\left(p^{2}\right)$ in the chiral expansion (this should be improved by a more accurate calculation at higher orders); iii) the vacuum polarization $\delta_{n}^{\mathrm{vac}}$ can be taken from $[11], \delta_{n}^{\mathrm{vac}} \simeq 1 \%$. We can now use Eq. (12) to compare the existing bound state data with predictions based on scattering data, that is taking the values for $a_{0,1}$ from various analyses of kaon-nucleon scattering data and calculate the strong shift and width with these values. In Fig. 2 we show the recent result from DEAR [12] and older results of the energy shift from kaonic hydrogen experiments in comparison with the predictions for $\Delta E$ and $\Gamma$ based on various sets of scattering lengths $a_{0}, a_{1}$ available in the literature 
$[13,14,15,16]$. As we immediately observe from the plot, the use of the lowest-order Deser formula, Eq. (8), (represented by the filled circles) can not be justified any more: both the cusp effect (as represented by the open circles) and Coulomb corrections have a size comparable with the present precision of the DEAR experiment, and should be taken into account in the extraction of the S-wave $K N$ scattering lengths from the experimental data. The figure also reveals that the recent DEAR data apparently are not consistent with the (older) scattering data. On the other hand, most predictions based on the scattering data are seen to be consistent with the older KEK kaonic hydrogen data [17]. I consider it premature to completely revise the low-energy antikaon-proton interaction based on the DEAR result but rather more refined studies on the accuracy of the extraction of the scattering lengths from the cross sections and mass distributions in $K^{-} p \rightarrow M B$ are called for. In addition, more precise data on e.g. $\pi \Sigma$ mass distributions would be very much needed to further sharpen the extraction of the $\bar{K} N$ scattering amplitude.

To summarize this part, we derived the formal expression for the strong shifts of the energy levels in kaonic hydrogen in QCD, up-to-and-including $O\left(\delta^{4}\right)$ in the isospin breaking parameter $\delta \sim \alpha, m_{d}-m_{u}$. The use of the non-relativistic effective Lagrangian approach allows one to treat that otherwise extremely complicated problem with a surprising ease. We discover that large isospin-breaking corrections arise, in particular, due to the following sources: (a) $s$-channel rescattering with the $\bar{K}^{0} n$ intermediate state (cusp effect), and (b) Coulomb corrections that are non-analytic in $\alpha$. We further prove that the remaining corrections are analytic in $\delta$ at $O(\delta)$ (for details see [9]). Examining some of these corrections, on the other hand, we do not find a big effect - the obtained values are at the percent level, which one expects to be a typical size of isospin breaking in QCD. The present status of corrections in kaonic hydrogen can be summarized by the Eq. (12). Instead of the combination $\frac{1}{2}\left(a_{0}+a_{1}\right)$ which enters in the original Deser formula (8), we propose to focus on the extraction of the quantity $\mathcal{T}_{K N}^{(0)}$ from the experimental data. The reason for this is that $\mathcal{T}_{K N}^{(0)}$ already includes the dominant non-analytic corrections in a parameter-free form. The remaining analytic corrections at $O(\delta)$ are contained in the quantities $\delta \mathcal{T}_{K N}$ and $\delta_{n}^{\text {vac }}$. The evaluation of $\delta \mathcal{T}_{K N}$ within CHPT could be interesting, but possibly complicated due to the expansion in the strange quark mass. At the present stage, in the absence of such calculations, the best is to include $\delta \mathcal{T}_{K N}$ in the estimate of the systematic error. From the above discussion one may hope that the effect from $\delta \mathcal{T}_{K N}$ should not exceed a few percent, which is a natural size of electromagnetic corrections. For further discussions of kaonic hydrogen and related issues, see the talks by Borasoy, Fayfman, Ivanov, Weise and Zmeskal at this conference.

\section{On the $K^{-} \alpha$ scattering length and antikaon-nuclear bound states}

The interest in exotic few-nucleon systems involving the $\bar{K}$-meson as a constituent was triggered by the work of [18] (and others) as well as the detection of a strange tribaryon $S^{0}(3115)$ in the interaction of stopped $K^{-}$-mesons with ${ }^{4} \mathrm{He}$ [19]. In this context, one 
needs to know the $K^{-} \alpha$ scattering length. In [20], we have addressed this issue and the possible consequences for antikaon-nuclear bound states.

To calculate the S-wave $K^{-} \alpha$ scattering length as well as the final-state-interaction (FSI) enhancement factor, we use the Foldy-Brueckner adiabatic approach based on the multiple scattering (MS) formalism [21]. In this framework, the continuum $K^{-} \alpha$ wave function, which is defined at fixed coordinates of the four nucleons in ${ }^{4} \mathrm{He}$, can be written as the sum of the incident plane wave of the kaon and waves emerging from the four fixed scattering centers. Keeping only the S-wave contribution, we can express the total wave function $\Psi_{k}$ through the $j$-channel wave functions $\psi_{j}\left(\mathbf{r}_{j}\right)$ in the following way

$$
\Psi_{k}\left(\mathbf{r}_{K^{-}} ; \mathbf{r}_{1}, \mathbf{r}_{2}, \mathbf{r}_{3}, \mathbf{r}_{4}\right)=\mathrm{e}^{i \mathbf{k} \cdot \mathbf{r}}+\sum_{j=1}^{4} t_{K^{-} N_{j}} \frac{\mathbf{e}^{i q R_{j}}}{R_{j}} \psi_{j}\left(\mathbf{r}_{j}\right),
$$

with $m\left(m_{K^{-}}\right)$the nucleon (charged kaon) mass, $R_{j}=\left|\mathbf{r}_{K^{-}}-\mathbf{r}_{j}\right|$ and the t-matrix, $t_{K^{-} N_{j}}$, is related to the elastic scattering amplitude $f_{K^{-} N}$ via $t_{K^{-} N}(k)=\left(1+m_{K^{-}} / m\right) f_{K^{-} N}(k)$, where $k$ is the modulus of the relative $\bar{K} N$ momentum. The S-wave $K^{-} \alpha$ scattering length can be derived from the asymptotic expansion of Eq. (13) at $r_{K^{-}} \rightarrow \infty$ and it is given by

$$
a_{K^{-\alpha}}=\left.\frac{m_{\alpha}}{m_{\alpha}+m_{K^{-}}}\left\langle\sum_{j=1}^{4} t_{K^{-N}} \psi_{j}\left(\mathbf{r}_{j}\right)\right\rangle\right|_{\sum_{j=1}^{4} \mathbf{r}_{j}=0}
$$

with $m_{\alpha}$ the $\alpha$-particle mass. Further, to obtain the FSI enhancement factor we calculate the total wave function $\Psi_{k}$ given by Eq. (13) at $\mathbf{r}_{K^{-}}=\sum_{j=1}^{4} \mathbf{r}_{j}=0$ and average it over the coordinates of the nucleons $\mathbf{r}_{j}$ in ${ }^{4} \mathrm{He}$. Thus the FSI enhancement factor is [21]

$$
\lambda^{\mathrm{MS}}\left(k_{K^{-} \alpha}\right)=\left|\left\langle\Psi_{q_{K^{-}}^{\text {lab }}}\left(\mathbf{r}_{K^{-}}=\sum_{j=1}^{4} \mathbf{r}_{j}=0 ; \mathbf{r}_{1}, \mathbf{r}_{2}, \mathbf{r}_{3}, \mathbf{r}_{4}\right)\right\rangle\right|^{2} .
$$

The integration in Eq. (15) over the nucleon coordinates $\mathbf{r}_{j}$ was performed using the Monte-Carlo method. This approach provides us with the possibility to include all configurations of the nucleons in ${ }^{4} \mathrm{He}$. Within this method we can also take into account in Eq. (13) the dependence of the $t_{K^{-} N_{j}}$ amplitude on the type of nucleonic scatterer, i.e. proton or neutron. The basic uncertainties of calculations in the MS approximation are given by the next-to-leading order model corrections such as recoil corrections, contributions from double and and triple scattering terms, etc and due to the uncertainties of the elementary $I=0$ and $I=1 \bar{K} N$ scattering lengths. The calculations of the $K^{-} \alpha$ scattering length were therefore done for five sets of parameters for the $\bar{K} N$ lengths shown in the Table 1. Unitarizing the constant scattering length, we can reconstruct the $\bar{K} \alpha$ scattering amplitude within the zero range approximation. We found a loosely bound state with binding energy $E_{R}=-2 \ldots-7 \mathrm{MeV}$ and width $\Gamma_{R}=11 \ldots 18 \mathrm{MeV}$. Our result differs from the prediction of Akaishi and Yamazaki [18] obtained under the assumption of a strongly attractive phenomenological $\bar{K} N$ potential but using the same scattering lengths of Set 1 as employed here. It is not clear if medium effects and higher order corrections might 


\begin{tabular}{|l|c|l|l|l|}
\hline Set & Reference & $a_{0}(K N)[\mathrm{fm}]$ & $a_{1}(K N)[\mathrm{fm}]$ & $a\left(K^{-} \alpha\right)[\mathrm{fm}]$ \\
\hline 1 & {$[22]$} & $-1.59+i 0.76$ & $0.26+i .57$ & $-1.80+i 0.90$ \\
\hline 2 & {$[22]$} & $-1.61+i 0.75$ & $0.32+i 0.70$ & $-1.87+i 0.95$ \\
\hline 3 & {$[23]$} & $-1.57+i 0.78$ & $0.32+i 0.75$ & $-1.90+i 0.98$ \\
\hline 4 & {$[24]$} & $-1.03+i 0.95$ & $0.94+i 0.72$ & $-2.24+i 1.58$ \\
\hline 5 & {$[15]$} & $-1.31+i 1.24$ & $0.26+i 0.66$ & $-1.98+i 1.08$ \\
\hline
\end{tabular}

Table 1: The $K^{-} \alpha$ scattering length for various sets of the $\bar{K} N$ scattering lengths $a_{0,1}$.
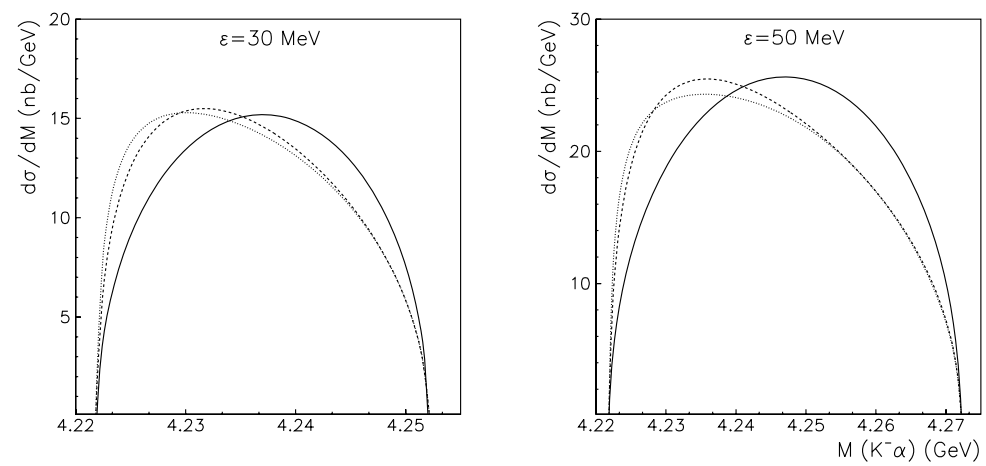

Figure 3: The invariant $K^{-} \alpha$ mass spectra produced in the $d d \rightarrow \alpha K^{+} K^{-}$reaction at excess energies 30 and $50 \mathrm{MeV}$. The solid lines describe the pure phase space distribution, while the dashed and dotted lines show our calculations with $K^{-} \alpha$ FSI given by parameters of Set 1 and 4 , respectively.

be so strong in order to change so drastically the $\bar{K} \alpha$ scattering length predicted by our calculations within the multiple scattering approach. In any case it is very important to measure the S-wave $\bar{K} \alpha$ scattering length and to clarify the situation concerning the possible existence of a (deeply) bound $\bar{K} \alpha$ state.

We have also analyzed the $K^{-} \alpha$ FSI in the reaction $d d \rightarrow \alpha K^{+} K^{-}$and discussed the possibility to evaluate the $K^{-} \alpha$ scattering length from the $K^{-} \alpha$ invariant mass spectra [20]. For that, we calculated the $K^{-} \alpha$ invariant mass spectra at excess energies $\epsilon=30$ and $50 \mathrm{MeV}$ which are shown in Fig. 3. The solid lines show the calculations for the pure phase space, i.e. assuming a constant production amplitude and neglecting FSI. The dashed and dotted lines in Fig. 3 show the results obtained with the $K^{-} \alpha$ FSI calculated with the parameters of the Set 1 and 4, respectively. All lines at each figure are normalized to the same value, given by the reaction cross section at a certain excess energy. At $\epsilon=50 \mathrm{MeV}$ the invariant mass spectra are normalized to the $d d \rightarrow \alpha K^{+} K^{-}$cross section of $1 \mathrm{nb}$. It is clear that the FSI significantly changes the $K^{-} \alpha$ mass spectra. The most pronounced effect is observed at low invariant masses available in the first $10 \mathrm{MeV}$ bin. To draw qualitative conclusions, one can compare the ratio of the cross sections at 
the lowest $K^{-} \alpha$ invariant masses, i.e. within the first $10 \mathrm{MeV}$ bin, calculated with and without FSI. We found that this ratio $R=1.26 \ldots 1.34$ at $\epsilon=30 \mathrm{MeV}, 1.49 \ldots 1.56$ at $\epsilon=50$ $\mathrm{MeV}$ and $1.84 \ldots 2.18$ at $\epsilon=100 \mathrm{MeV}$. Here the limits of the ratio at each excess energy are given by the calculations with the $\bar{K} N$ scattering length from the Set 1 and Set 4 . With these estimates it is clear a that reasonable determination of the $K^{-} \alpha$ scattering length requires high statistical measurements at $K^{-} \alpha$ invariant masses below $4.23 \mathrm{GeV}$, i.e. approximately 100 events. Such a high precision experiment apparently can be done at the Jülich Cooler Synchrotron COSY. This shows that the measurement of the $K^{-} \alpha$ mass distribution near the reaction threshold may provide a new tool for the determination of the $s$-wave $K^{-} \alpha$ scattering length. It is important to stress that for kaonic helium atoms, energy shifts can be measured for the $2 p$ state and widths for the $2 p$ and $3 d$ states. The $n p \rightarrow 1 s$ transitions for ${ }^{4} \mathrm{He}$ cannot be observed since the absorption from the $p$ states is almost complete. Therefore the possibility to determine the S-wave $\bar{K} \alpha$ scattering length from experiments with kaonic atoms is questionable. With this respect a measurement at COSY provides an unique opportunity to determine the S-wave $K^{-} \alpha$ scattering length. Furthermore, we have investigated the momentum dependence of the enhancement factor $\lambda^{\mathrm{MS}}(k)$ calculated within the multiple scattering approximation and compared it with the one obtained utilizing the Watson-Migdal formalism. It was found that the absolute difference between both calculations is relatively small at momenta $q \leq 100 \mathrm{MeV} / \mathrm{c}$. Fur further discussions of antikaonic bound states, see the talks by Akaishi, Dote, Itahashi, Iwasaki, Nagae, Suzuki, Weise and Yamazaki at this conference.

\section{Summary and outlook}

Hadronic atoms allow one to access meson-baryon scattering lengths, leading to stringent tests of the properties of QCD in the non-perturbative regime. In addition, exotic nuclear systems with bound antikaons give further constraints on the so important $\bar{K} N$ interaction. On the theoretical side, isospin breaking in pionic deuterium and kaonic deuterium should be studied along the lines outlined here. In addition, more systematic studies of exotic nuclei with antikaons as constituents are called for.

\section{Acknowledgements}

First, it is a great pleasure to thank Akaki Rusetsky and Udit Raha for a most enjoyable collaboration in Bonn. I also thank my Jülich, INR Moscow and ITEP collaborators and the organizers for a very pleasant meeting.

\section{References}

[1] U.-G. Meißner, U. Raha and A. Rusetsky, Eur. Phys. J. C 41 (2005) 213 [nuclth/0501073].

[2] P. Hauser et al., Phys. Rev. C 58 (1998) 1869.

[3] S.R. Beane and M.R. Savage, Nucl. Phys. A 717 (2003) 104 [nucl-th/0204046]. 
[4] S. R. Beane, V. Bernard, E. Epelbaum, U.-G. Meißner and D. R. Phillips, Nucl. Phys. A 720 (2003) 399 [hep-ph/0206219].

[5] P. Zemp, in Proceedings of "HadAtom03" Workshop, 13-17 October 2003, ECT* (Trento, Italy), arXiv:hep-ph/0401204; J. Gasser and P. Zemp, in preparation.

[6] P. Büttiker et al., in progress.

[7] B. Borasoy and H. W. Grießhammer, Int. J. Mod. Phys. E 12 (2003) 65 [nuclth/0105048].

[8] D. Gotta, Int. J. Mod. Phys. A 20 (2005) 349.

[9] U.-G. Meißner, U. Raha and A. Rusetsky, Eur. Phys. J. C 35 (2004) 349 [hep$\mathrm{ph} / 0402261]$.

[10] R.H. Dalitz and S.F. Tuan, Ann. Phys. 3 (1960) 307.

[11] D. Eiras and J. Soto, Phys. Lett. B 491 (2000) 101 [hep-ph/0005066].

[12] C. Guaraldo et al., Eur. Phys. J. A 19 (2004) SUPPL1185.

[13] A.D. Martin, Nucl. Phys. B 179 (1981) 33.

[14] E. Oset and A. Ramos, Nucl. Phys. A 635 (1998) 99 [nucl-th/9711022].

[15] U.-G. Meißner and J.A. Oller, Phys. Lett. B 500 (2001) 263 [hep-ph/0011146].

[16] B. Borasoy, R. Nißler and W. Weise, arXiv:hep-ph/0410305.

[17] T.M. Ito et al., Phys. Rev. C 58 (1998) 2366.

[18] Y. Akaishi and T. Yamazaki, Phys. Rev. C 65 (2002) 044005.

[19] T. Suzuki et al., Phys. Lett B 597 (2004) 263.

[20] V. Y. Grishina, L. A. Kondratyuk, A. Sibirtsev, M. Büscher, S. Krewald, U.G. Meißner and F. P. Sassen, arXiv:nucl-th/0503076.

[21] M. L. Goldberger and K. M. Watson, Collision Theory, John Wiley and Sons, Inc., New York, London, Sidney (1964).

[22] R. C. Barrett, A. Deloff, Phys. Rev. C 60 (1999) 025201.

[23] R. H. Dalitz, T. C. Wong, and G. Rajasekaran, Phys. Rev. 153 (1967) 1617.

[24] J.E. Conboy, Rutherford-Appleton Lab. Report, RAL-85-091, 1985. 
